\title{
CONSTRUÇÃO E VALIDAÇÃO DE UM MANUAL DE DETECÇÃO DO PÉ DIABÉTICO PARA ATENÇÃO PRIMÁRIA
}

José William Araújo do Nascimento' Edjôse Ciriaco Santana Silva ${ }^{1}$ Manoel Luiz Ferreira Junior ${ }^{1}$ Suzane Brust de Jesus ${ }^{1}$

\author{
https://orcid.org/0000-0002-1844-1117 \\ https://orcid.org/0000-0002-0820-1435 \\ https://orcid.org/0000-0003-0427-5493 \\ https://orcid.org/0000-0002-3826-8200
}

Objetivo: descrever o processo de construção e validação de um manual de detecção do pé diabético para os profissionais de saúde da Atenção Primária. Metodologia: pesquisa metodológica, realizada em 2019, seguindo as etapas: levantamento bibliográfico, construção do manual, validação do conteúdo pelos juízes, adequação do manual e diagramação. Para avaliação da confiabilidade interna, um questionário foi respondido por 20 juizes. Posteriormente foi calculado o Índice de Validade de Conteúdo de cada um dos itens (IVC). A validade de conteúdo dos itens neste estudo foi considerada quando IVC $\geq 0,75$, conforme referencial metodológico. Resultados: o manual foi construido e na avaliação geral, obteve IVC com valores acima de 0,75 e IVC total de 0,85. Conclusão: o manual apresentou confiabilidade e fidedignidade para observação da qualidade na conduta preventiva do pé diabético na Atenção Primária. No entanto exige outras fases para uma validação de conteúdo mais precisa e específica.

Descritores: Atenção Primária à Saúde; Estudos de Validação; Pé Diabético.

\section{CONSTRUCTION AND VALIDATION OF A PREVENTIVE MANUAL OF DIABETIC FOOT IN PRIMARY CARE}

Objective: the article describes the steps in producing and validating an diabetic foot detection manual for primary care health professionals. Methodology: methodological study conducted in 2019, consisting of the following steps: bibliographic survey, manual construction, content validation by judges, adequacy of the manual and layout. To assess internal reliability, a questionnaire was answered by 20 judges. Subsequently, the Content Validity Index of each item (CVI) was calculated. The content validity of the items in this study was considered when CVI $\geq 0.75$, according to the methodological framework. Results: the preventive manual was constructed and in the general evaluation, CVI with values above 0.75 and total CVI of 0.85 . Conclusion: the manual presented for observing the quality of preventive management of diabetic foot in Primary Care. However it requires other phases for more accurate and specific content validation.

Descriptors: Primary Health Care; Validation Studies; Diabetic Foot.

\section{CONSTRUCCIÓN Y VALIDACIÓN DE UN MANUAL PREVENTIVO DE PIE DIABÉTICO EN ATENCIÓN PRIMARIA}

Objetivo: describir el proceso de construcción y validación de un manual de prevención y detección del pie diabético para profesionales de la salud de atención primaria. Metodologia: investigación metodológica, realizada en 2019, siguiendo las etapas: estudio bibliográfico, construcción manual, validación de contenido por jueces, adecuación del manual y diseño. Para evaluar la confiabilidad interna, 20 jueces respondieron un cuestionario. Posteriormente, se calculó el Índice de validez de contenido de cada elemento (CVI). La validez del contenido de los ítems en este estudio se consideró cuando CVI $\geq 0.75$, de acuerdo con el marco metodológico. Resultados: se construyó el manual y en la evaluación general, CVI con valores por encima de 0.75 y CVI total de 0.85. Conclusión: el manual presentó confiabilidad y confiabilidad para observar la calidad del manejo preventivo del pie diabético en Atención Primaria. Sin embargo, requiere otras fases para una validación de contenido más precisa y específica.

Descriptors: Atención Primaria de Salud; Estudios de Validación; Pie Diabético.

IUniversidade Católica de Pernambuco

Autor Correspondente: José William Araújo do Nascimento - Email: jwan10@hotmail.com.br 


\section{INTRODUÇÃO}

O Diabetes Mellitus (DM) está entre as doenças crônicas de maior destaque no cenário da saúde, tanto a nível nacional quanto ao global. Em 2014, estimativas da apontaram 422 milhões de adultos com DM, o que equivale a quatro vezes mais a quantidade de pessoas com esta patologia em 1980 (108 milhões) $)^{(1)}$.

No cenário brasileiro, no ano de 2017, havia mais de 13 milhões de pessoas com DM, levando a um custo anual de aproximadamente 24 milhões de dólares com despesas no setor de saúde relacionadas à doença ${ }^{(2)}$.

As complicações nos pés são uma das principais causas de morbimortalidade em pessoas que têm diabetes, contribuindo desta forma para o aumento do uso e dos custos com a saúde (3-4).

Esta doença quando não monitorada de forma oportuna, traz complicações evidentes como o pé diabético (PD). Este termo é utilizado para designar as diversas lesões que podem ocorrer no pé do indivíduo com diabetes. Se caracteriza por lesões cutâneas e profundas relacionadas a alterações neuropáticas, vasculares, ortopédicas, infecciosas e funcionais ${ }^{(5)}$.

Conforme pesquisadores, estima-se que até um terço das pessoas diagnosticadas com diabetes irão desenvolver uma úlcera no pé. Ulcerações não cicatrizantes ou crônicas podem levar à infecção e subsequente amputação(6).

O panorama epidemiológico, social e econômico que o DM vem apresentando, demonstra a necessidade da implementação de políticas públicas de saúde voltadas ao usuário e que propiciem prevenção dos agravos e melhoria da qualidade de vida(5).

É principalmente na Atenção Primária onde os profissionais de saúde devem desenvolver estratégias que permitam intervenções que venham a contribuir para o tratamento eficaz e prevenção de complicações( ${ }^{(7)}$.

A utilização de manuais na Atenção Primária é uma das estratégias que pode ser utilizada pelos profissionais de saúde para a orientação e detecção de situações específicas $^{(8)}$.

Estes manuais facilitam o trabalho da equipe multidisciplinar tanto na orientação de usuários e familiares no processo de tratamento, recuperação e autocuidado como na detecção precoce de patologias e suas complicações. Entretanto, devem ter uma linguagem clara e objetiva para os indivíduos que o utilizarão e desta forma, deve-se validar seu conteúdo(9-10).

Frente a este contexto, visando sistematizar o atendimento ao usuário diabético, o objetivo do presente estudo foi construir e validar um manual de prevenção e detecção

do pé diabético para profissionais de saúde da Atenção Primária.

\section{METODOLOGIA}

Trata-se de uma pesquisa metodológica que tem como objetivo o desenvolvimento, a avaliação e o aperfeiçoamento de instrumentos e estratégias metodológicas $^{(11)}$

O presente estudo foi realizado durante o ano de 2019 e teve como foco o desenvolvimento de um manual, a ser utilizado em estratégias de saúde da família durante o acompanhamento de usuários diabéticos, para promover a prevenção e detecção precoce de casos de pé diabético. O processo de construção do manual foi adaptado às premissas para a elaboração de manuais de orientação para o cuidado em saúde ${ }^{(8)}$.

Figura 1: Etapas seguidas no desenvolvimento do manual informativo, Recife, PE, Brasil, 2019.

\section{Construção}

1. Levantamento

bibliográfico;

\section{Seleção e fichamento} do conteúdo;

3. Elaboração textual;

4. Criação das ilustrações

(figuras, quadros

e algoritmos)

\section{Diagramação}

Após o levantamento bibliográfico foram utilizados 21 artigos científicos, 09 livros-textos e 03 manuais do Ministério da Saúde ${ }^{(3,5,12)}$. A busca desse material foi guiada por uma ou mais das seguintes palavras-chaves: diabetes mellitus, pé diabético, úlcera de pé diabético, úlcera venosa e prevenção.

A finalidade desta seleção foi reunir todo o conteúdo de interesse para o manual informativo, sendo categorizados nos seguintes grupos: conceito de diabetes e pé diabético; exame físico; orientação de autocuidado.

A elaboração das ilustrações foi baseada na leitura reflexiva, ou seja, a partir do referencial literário fichado e dos principais conteúdos abordados no manual. Dois algoritmos foram elaborados com o objetivo de oferecer um plano de condutas que possam detectar precocemente o pé diabético na Atenção Primária. 
Para a confiabilidade interna do manual, foi realizada consulta a juízes (especialistas na área) entre os meses de fevereiro a abril (2019). Como critérios de inclusão dos juízes, participaram do estudo aqueles que eram portadores de certificado de curso de graduação em Enfermagem ou Medicina.

Os especialistas foram selecionados por meio da amostragem bola de neve ${ }^{(11)}$. Após a indicação, era realizada consulta ao Currículo Lattes para verificar a adequação do juíz aos critérios de seleção para esse estudo.

Participaram do estudo juízes atuantes na Atenção Primária e especializada, bem como, os especialistas em pé diabético (endocrinologista, cirurgião vascular e estomaterapeuta).

Mediante o envio de uma carta convite e do Termo de Consentimento Livre Esclarecido (TCLE), 20 profissionais responderam a um questionário que abordara aspectos inerentes as suas formações acadêmicas-profissional e a aspectos sobre o instrumento de avaliação do pé diabético elaborado; este último abordou temas como facilidade de leitura, vocabulário, sequência do material educativo, descrições e orientações abordadas pelo material. $\bigcirc$ juiz dispunha de um espaço para futuras sugestões de melhorias do instrumento.

Em relação a análise estatística dos itens referentes ao caráter acadêmico-profissional dos juizes, utilizou-se no SPSS (Statistical Package for Social Science) o Teste de Qui-Quadro, para o respectivo fim.

Após a avaliação do manual, procedeu-se a validação de conteúdo. Para esta, foi utilizado o Índice de Validade de Conteúdo (IVC), que afere a concordância dos juízes quanto à representatividade de determinados aspectos do instrumento e de seus itens.

Este método consiste de uma escala de Likert com pontuação de 1 a 4, em que: 1 (item não equivalente); 2 (item necessita de grande revisão para ser avaliada a equivalência); 3 (item equivalente, necessita de pequenas alterações); e 4 (item absolutamente equivalente). Para o cálculo do IVC de cada item do questionário, basta somar as respostas 3 e 4 dos participantes do comitê de especialistas e dividir o resultado desta, pelo número total de respostas ${ }^{(13)}$

De modo a estipular a taxa de concordância aceitável entre os juízes, foram estabelecidos os valores recomendados de no mínimo 0,75 entre eles para servirem de critério de decisão sobre a pertinência e/ou aceitação do item.

Após essas análises, foram realizadas as alterações necessárias, de acordo com a pontuação final, no que diz respeito aos itens com baixos escores. Além disso, também foram apreciadas as observações e sugestões dos juízes, quanto à necessidade de mudanças no material.
Registra-se que esse estudo seguiu a Resolução $n$. 466/2012 ${ }^{(14)}$ e foi aprovado por Comitê de Ética em Pesquisa, sob o CAAE 65743417.8.0000.5206

\section{RESULTADOS}

\section{O processo de construção do manual}

Na elaboração textual, os autores procuraram organizar as informações de maneira a retratar todo o percurso de avaliação do pé do indivíduo com diabetes bem como as orientações fornecidas a eles. Os tópicos abordados no manual foram: conceituação do diabetes mellitus e pé diabético; estratificação de risco para o pé diabético; fisiopatologia do pé diabético; classificação do pé diabético; avaliação física dos pés do usuário diabético; avaliação cutânea; avaliação dos calçados; avaliação musculoesquelética; avaliação vascular; avaliação neurológica periférica; instrução de autocuidado aos usuários diabéticos.

Ao final, o manual foi composto por 28 páginas e 16 ilustrações. Foram desenvolvidos dois algoritmos de condutas para detecção do pé diabético na Atenção Primária. Um se referia a todos exames físicos e outro apenas a avaliação neurológica. A Figura 2 apresenta a diagramação de alguns tópicos do manual.

Figura 2: Diagramação de alguns tópicos do manual, Recife, PE, Brasil, 2019.

\section{AVALIAÇÃO DOS CALÇADOS}

Para começar, avalie o formato e a adaptação, e a seguir observe osmateriais (couroou plástico) de que são feitos a parte superiore inferior do calçado de seu usuário diabético. Faça uma revisão dos sapatos, para remover qualquer objeto estranho, e a seguir introduza a mão na ponta deste, para buscar quaisquer irregularidades como projeção das costuras para dentro, dobras ou desgaste do revestimento. Remova as palmilhas e busque por expressões ocasionadas por saliências ósseas.
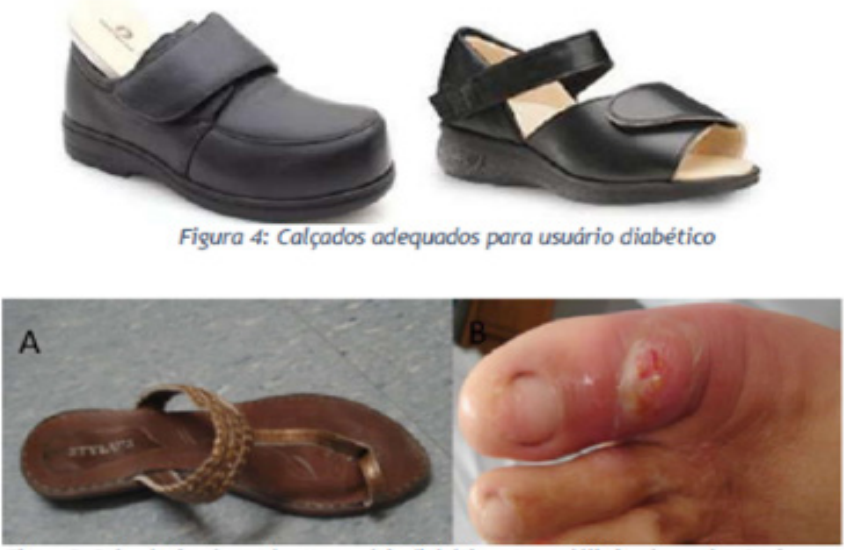

Fiģura 5: Calçados inadequados para usário diabético - A (sandália inodequada), B (abcesso no dorso do hálux, causada pela sandália A). Fonte: CAIAFA, et al., 2011. 


\section{AVALIAÇÃOVASCULAR}

Durante a avaliação vascular dos membros inferiores é importante que o profissional de saúde esteja atento às alterações como rubor em declive, varizes, edema e pele fina e brilhante.

É de fundamental importância a realização da palpação dos pulsos tibial posterior (PTP) e pedioso (PP), uma vez que estes podem estar normais, diminuídos ou ausentes.
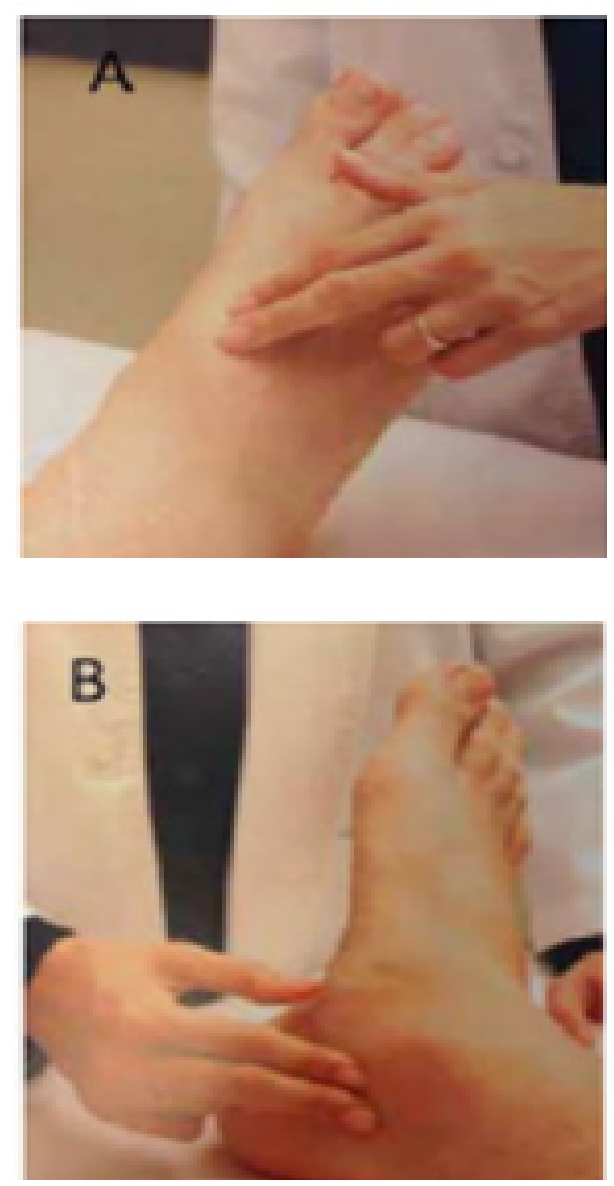

Figura 7: Avaliaçäos dos pulsos podálicos - A (palpoçōo do PP) 8 (polpoçäo do PTP). Fonte: BRASIL, 2016 .

Nesta mesma avaliação, esteja atento aos sinais clássicos de: Isquemia aguda: Dor, paralisia, parestesia, ausência de pulso, paralisia por frio e palidez (BRASIL, 2016).

Isquemia critica: Dor na perna em repouso, gangrena, feridas/ úlceras que não cicatrizam, atrofia muscular, rubor depedente, palidez quando a perna é elevada, rarefação dos pelos sobre odorso do pé, unhas espessas e pele brilhante (BRASIL, 2016; IWGDF, 2019; SANTOS, et al., 2015).

\section{Consulta a especialistas da área de interesse}

Quanto ao perfil dos juízes, houve predominância do sexo feminino (55\%). Em relação a idade, $40 \%$ possuía entre 35 e 44 anos. A maioria (30\%) tinha entre 8 a 12 anos de formado.
Tabela 1: Caracterização dos juízes participantes da pesquisa. Recife, PE, Brasil, 2019.

\begin{tabular}{|c|c|c|c|c|}
\hline Tipo de graduação & n & $\%$ & $\%$ acumulado & Valor do $p$ \\
\hline Enfermagem & 10 & 50,0 & 50 & \\
\hline Medicina & 10 & 50,0 & 100 & 0,591 \\
\hline Total & 20 & 100,0 & & \\
\hline Maior titulação & $\mathrm{n}$ & $\%$ & $\%$ acumulado & Valor do $p$ \\
\hline $\begin{array}{l}\text { Residência/ espe- } \\
\text { cialista }\end{array}$ & 7 & 35,0 & 35,0 & \\
\hline Mestre & 7 & 35,0 & 70,0 & 0,031 \\
\hline Doutor & 6 & 30,0 & 100,0 & \\
\hline Total & 20 & 100,0 & & \\
\hline $\begin{array}{l}\text { Experiência no en- } \\
\text { sino }\end{array}$ & $\mathrm{n}$ & $\%$ & $\%$ acumulado & Valor do $\mathrm{p}$ \\
\hline 1 a 5 anos & 3 & 15,0 & 15,0 & \\
\hline 6 a 10 anos & 6 & 30,0 & 45,0 & \\
\hline \multirow[t]{2}{*}{11 a 15 anos } & 1 & 5,0 & 50,0 & \\
\hline & & & & 0,301 \\
\hline Acima de 16 anos & 8 & 40,0 & 90,0 & \\
\hline Não possui & 2 & 10,0 & 100,0 & \\
\hline Total & 20 & 100,0 & & \\
\hline
\end{tabular}

$\begin{array}{lcccc}\begin{array}{l}\text { Experiência na as- } \\ \text { sistência }\end{array} & \text { n } & \% & \% \text { acumulado } & \text { Valor do } p \\ 1 \text { a } 5 \text { anos } & - & - & - & \\ 6 \text { a } 10 \text { anos } & 5 & 25,0 & 25,0 & 0,439 \\ 11 \text { a } 15 \text { anos } & 4 & 20,0 & 45,0 & \\ \text { Acima de } 16 \text { anos } & 11 & 55,0 & 100,0 & \\ \text { Total } & & & & \end{array}$

Teste Qui-quadrado de independência. *Nível de significância $p>0,05$.

Referente a caracterização dos juizes que participaram da confiabilidade interna, a tabela 1 demonstra que 10 (50\%) eram profissionais de enfermagem; 20 (100\%) declararam terem residência/especialização; 8 (40\%) possui mais de 16 anos de experiência no ensino e 11 
(55\%) declarou ter acima de 16 anos de experiência na assistência.

Dos 20 juízes que participaram desta etapa, 70\% eram do estado de Pernambuco enquanto que os 30\% restantes eram de estados como Amazonas, Goiás, Minas gerais, São Paulo, Santa Catarina e Rio de Janeiro.

\section{Validação de conteúdo}

Tabela 2: Julgamento entre os juízes acerca do manual de prevenção do pé diabético. Recife, PE, Brasil, 2019.

\begin{tabular}{|c|c|c|c|c|c|}
\hline \multirow[t]{2}{*}{ Variável } & \multicolumn{2}{|c|}{$\begin{array}{l}\text { Adequado } \\
\text { (escore 4) }\end{array}$} & \multicolumn{3}{|c|}{$\begin{array}{l}\text { Adequado } \\
\text { com alterações } \\
\text { (escores } 2 \text { e 3) }\end{array}$} \\
\hline & $\mathrm{n}$ & $\%$ & $n$ & $\%$ & IVC \\
\hline Apresentação gráfica & 10 & 50,0 & 09 & 45,0 & 0,80 \\
\hline Facilidade de leitura & 12 & 60,0 & 08 & 40,0 & 0,90 \\
\hline Vocabulário & 12 & 60,0 & 08 & 40,0 & 0,90 \\
\hline Sequência & 15 & 75,0 & 04 & 20,0 & 0,85 \\
\hline $\begin{array}{l}\text { Características gráficas } \\
\text { dos quadros }\end{array}$ & 10 & 50,0 & 09 & 45,0 & 0,75 \\
\hline $\begin{array}{l}\text { Condutas terapêuticas } \\
\text { do algoritmo } 1\end{array}$ & 10 & 50,0 & 09 & 45,0 & 0,85 \\
\hline $\begin{array}{l}\text { Condutas terapêuticas } \\
\text { do algoritmo } 2\end{array}$ & 14 & 70,0 & 04 & 20,0 & 0,90 \\
\hline
\end{tabular}

A Tabela 2 representa o julgamento em relação as variáveis/itens que compõem o manual de prevenção do pé diabético e demonstra que nenhum deles foi avaliado como inadequado e todos obtiveram concordância dentro do nível estabelecido (IVC $\geq 0,75$ ). Em relação ao IVC, todos os itens atingiram níveis acima de 0,75 e se obteve IVC total do instrumento de 0,85.

Foram 07 itens considerados adequados com alterações, dos quais cinco pertenciam a análise estrutural do material e dois relacionados a conduta terapêutica no manejo preventivo do pé diabético.

No Quadro 1, são apresentadas as sugestões realizadas pelos juízes para que os itens considerados adequados com alterações sejam reformulados e melhorados.
Quadro 1: Sugestões dos juízes acerca dos itens considerados adequados com alterações. Recife, PE, Brasil, 2019.

\begin{tabular}{ll} 
Requisitos avaliados & Sugestões dos juizes \\
\hline Apresentação gráfica & Deixar o material menos poluido \\
\hline Facilidade de leitura & --
\end{tabular}

Vocabulário

Quanto ao vocabulário, fácil entendimento, somente correções ortográficas.

Definir melhor a sequência do material de
forma que o leitor tenha uma condução
lógica

Características gráficas

Melhorar qualidade das palavras e ampliar resolução; mudar as cores nos quadros

uma vez que está dificultando a leitura.

dos quadros

$\begin{array}{ll}\text { Condutas terapêuticas do } & \text { Definir melhor as condutas e utilizar setas } \\ \text { algoritmo } 1 & \text { no algoritmo }\end{array}$

Condutas terapêuticas do

Definir melhor as condutas de modo que algoritmo 2

realmente seja um algoritmo ou fluxograma; inserir setas de uma conduta a outra

Na avaliação geral, o manual de prevenção e detecção do pé diabético obteve IVC com valores acima de 0,75, bem como apresentação de IVC total de 0,85, o que demonstra a alta confiabilidade e fidedignidade do instrumento para prevenir o pé diabético na Atenção Primária.

\section{DISCUSSÃO}

Considerando que o pé diabético é uma das principais complicações do DM, passiveis de forte intervenção na Atenção Primária, a elaboração do manual de prevenção e detecção é também, uma maneira de uniformizar o cuidado e oficializar as medidas preventivas, através das opiniões das diversas especialidades da área da saúde ${ }^{(15)}$.

Mediante conhecimento da fisiopatologia e etiologias do pé diabético, usuários de alto risco podem ser identificados precocemente, evitando feridas e/ou úlceras e amputações ${ }^{(16,17)}$. Conforme estudo, até $50 \%$ das amputações podem ser evitadas através do estímulo ao autocuidado e de um atendimento interdisciplinar baseado em evidências científicas ${ }^{(18)}$. 
Nesta perspectiva, a ideia da construção do manual informativo surgiu a partir das necessidades dos profissionais de saúde da Atenção Primária em prevenir e detectar o pé diabético precocemente.

O primeiro passo na elaboração do manual foi a busca na literatura especializada do conhecimento científico sobre a temática proposta. Assim, pode-se definir conceitos e cuidados importantes que, se seguidos, tem a capacidade de contribuir de forma eficaz na prevenção das complicações, para a melhoria das condutas terapêuticas e para a recuperação de usuários submetidos a diferentes tratamentos ${ }^{(19)}$.

Para um maior controle da avaliação do manual, especialistas das cinco regiões do Brasil, participaram do processo de confiabilidade interna, assegurando desta forma, as diferenças regionais que também permeiam os processos educativos e informativos ${ }^{(20)}$.

No que tange a confiabilidade interna, os primeiros itens que foram avaliados pelos participantes desta pesquisa, foram aqueles relacionados à classificação das características e dos seus conteúdos, que variou entre adequado (escore 4) e adequado com alterações (escores 2 e 3).

Estes itens são importantes para serem analisados pelos avaliadores, uma vez que o manual precisa ser atrativo; objetivo; não pode ser muito extenso, mas deve dar uma orientação significativa sobre o tema a que se propõe ${ }^{(8)}$

Por ser um material que precise de objetividade, foram criados dois algoritmos que contém condutas terapêuticas que visam a identificação precoce do pé diabético na Atenção Primária. Isto é um meio facilitador tanto para o usuário quanto para o profissional, pois se identificado alterações, o encaminhamento para os níveis de atenção especializada será imediato, evitando-se assim, maiores complicações como a amputação dos pés ${ }^{(19)}$.

Na prática clínica, o estabelecimento de uma linha de conduta padroniza é necessária pois tem a finalidade de adequar a assistência ao controle apropriado do pé diabético e suas implicações uma vez que assinala, de forma sistematizada, essa problemática na perspectiva intersetorial (19).

O direcionamento de condutas pode direcionar discussões e reflexões organizadas com os diferentes setores envolvidos na atenção à saúde, na busca de alternativas que visem a melhoria do atendimento ao diabético(21).
Neste limiar, as condutas presentes nos algoritmos 1 (avaliação geral) e 2 (avaliação neurológica periférica) receberam validação pertinentes, IVC 0,85 e 0,90 respectivamente.

No presente estudo, maior parte dos juizes concordou com a aplicabilidade do manual, considerando uma importante ferramenta que contém informações capazes de apoiar a sua decisão clínica no manejo de prevenção e detecção do pé diabético.

O processo de análise da confiabilidade interna do manual realizado pelos juizes obteve um IVC geral $(0,85)$. Desta forma, este estudo demonstrou que o manual de prevenção do pé diabético impresso e em meio eletrônico, fornecido aos profissionais da Atenção Primária a Saúde, contendo informações objetivas e claras do problema, foi um recurso efetivo para a melhoria do nível de informação e conduta, sobre o tema em questão.

Destaca-se que os manuais devem conter figuras, com a finalidade de facilitar a compreensão dos indivíduos que o utilizam ${ }^{(22)}$. A sequência do conteúdo, a organização, a fonte das letras e as características gráficas devem ser escolhidos adequadamente, para facilitar a sua leitura ${ }^{(23)}$.

Sendo de fácil utilização, o manual ajudará os profissionais que atendem usuários diabéticos nas Unidades de Saúde da família em suas práticas diárias.

\section{Contribuições para a prática}

A proposta de um manual para auxiliar a detecção do pé diabético para ser utilizado por profissionais de Enfermagem na Atenção Primária auxiliará a prestação da assistência de enfermagem segura e de qualidade para os portadores de lesões

\section{Limitações do estudo}

As limitações do estudo correspondem à necessidade da realização de outras fases do processo de validação, necessitando então, da ressubmissão do material aos juízes para reteste.

\section{CONCLUSÃO}

O manual de prevenção e detecção do pé diabético na Atenção Primária apresentou, por meio da avaliação dos juízes, confiabilidade e fidedignidade para observação da qualidade na conduta preventiva e de manejo do PD, entretanto, alguns itens necessitaram de alterações. 


\section{REFERÊNCIAS}

1. World Health Organization. Global report on diabetes. Geneva: WHO, 2016. Available from: https://apps.who.int/ iris/bitstream/handle/10665/204871/9789241565257_eng. pdf:jsessionid=D33B1381EBA3C4294D22CAF9AFECD787? sequence $=1$

2. International Diabetes Federation. IDF the IDF Diabetes Atlas. 8 ed. Brussels, Belgium. 2017. Available from: https://www.idf.org/ component/attachments/attachments.html?id=1405\&task=download 3. Ministério da Saúde (BR). Secretaria de Atenção à Saúde. Departamento de Atenção Básica. Estratégias para o cuidado da pessoa com doença crônica: diabetes mellitus. Brasilia, DF: Ministério da Saúde, 2013. 20-160 p. (Cadernos de Atenção Básica, n. 36). Available from: http://189.28.128.100/dab/docs/portaldab/ publicacoes/caderno_36.pdf

4. Mcewen NL, Ylitalo KR, Munson M, et al. Complicações e mortalidade nos pés: resultados da tradução da pesquisa em ação para o diabetes (TRIAD). J Am Podiatr Med Assoc. 2016[cited 2019 Jan 22]:106(1): 7-14.

5. Ministério da Saúde (BR). Manual do pé diabético: estratégias para - cuidado da pessoa com doença crônica. Brasília: Departamento de Atenção Básica. 2016[cited 2018 Dez 11]. Available from: http://189.28.128.100/dab/docs/portaldab/publicacoes/manual_do_ pe_diabetico.pdf

6. Armstrong DG, Boulton AJM, Bus SA. Úlceras do pé diabético e sua recorrência. N Engl J Med. 2017[cited 2019 Jan 21]: 376(24):2367-5. doi: https://doi.org/10.1056/NEJMral615439

7. Silveira GL, Ramos JLS, Freitas GLS, Rodrigues KL, Serafim SC, Brito RN, et al. Atuação do enfermeiro frente à adesão de idosos ao tratamento de diabetes. Revista e-ciencia. 2015[cited 2019 Fev 12]: 3(1):47-53. Available from: http://www.revistafjn.com.br/revista/index. php/eciencia/article/download/60/pdf_12

8. Echer IC. Elaboração de manuais de orientação para o cuidado em saúde. Rev Latino-am Enfermagem. 2005[cited 2019 Jan 11]:13(5):7547. Available from: http://www.scielo.br/pdf/rlae/v13n5/v13n5a22.pdf

9. Berardinell LM, Guedes NA, Ramos JP, Silva MG. Tecnologia educacional como estratégia de empoderamento de pessoas com enfermidades crônicas. Rev enferm UERJ. 2014[cited 2019 Jan 23]: 22(5):603-9. Available from: http://www.facenf.uerj.br/v22n5/ v22n5a04.pdf

10. Polit DF, Beck CT. Fundamentos da pesquisa em enfermagem: avaliação de evidências para a prática de enfermagem. 7̣a ed. Porto Alegre: ArtMed; 2011.

11. Ministério da Saúde (BR). Secretaria de Atenção à Saúde. Departamento de Atenção Básica. Diretrizes para o cuidado das pessoas com doenças crônicas nas redes de atenção à saúde e nas linhas de cuidado prioritárias. Brasilia: Ministério da Saúde; 2013. Available from: http://bvsms.saude.gov.br/bvs/publicacoes/ diretrizes\%20_cuidado_pessoas\%20_doencas_cronicas.pdf

12. Alexandre NMC, Coluci MZO. Validade de conteúdo nos processos de construção e adaptação de instrumentos de medidas. Ciência \& Saúde Coletiva. 2011[cited 2019 Mar 22]: 16(7):3061-8. Available from: http://www.scielo.br/pdf/csc/v16n7/06.pdf

13. Ministério da Saúde (BR). Resolução 466, Princípios éticos em pesquisa com seres humanos. 12 de dezembro de 2012. Available from: http://conselho.saude.gov.br/resolucoes/2012/Reso466.pdf

14. Silva MJ, Victor JF, Mota FRN, Soares ES, Leite BMB, Oliveira ET. Análise das propriedades psicométricas do APGAR de familia com idosos do nordeste brasileiro. Esc Anna Nery. 2014[cited 2019 Mai 17]:18(3):527-32. Available from: http://www.scielo.br/pdf/ean/ v18n3/1414-8145-ean-18-03-0527.pdf

15. Cunha JB, Salomé GM. Algoritmo e software para avaliação e tratamento de feridas. (Dissertação). Pouso Alegre (MG): Mestrado Profissional em Ciências Aplicada à Saúde, Universidade do Vale do Sapucaí. 2015. Available from: http://www.univas.edu.br/menu/ posgraduacao/MESTRADOS.asp

16. Menezes LCG, Guedes MVC, Moura NS, Oliveira RM, Vieira LA, Barros AA. Estratégias educativas para pessoas diabéticas com pé em risco neuropático: sintese de boas evidências. Rev. Eletr. Enf. 2016[cited 2019 Mai 1l]:18:el197. doi: http://dx.doi.org/10.5216/ree.v18.40281

17. Diretrizes da Sociedade Brasileira de Diabetes: 2015 - 2016. São Paulo: AC Farmacêutica, 2016. Available from: https://www.diabetes. org.br/profissionais/images/docs/DIRETRIZES-SBD-2015-2016.pdf

18. Amogne W, Reja A, Amare A. Diabetic foot disease in Ethiopian patients: a hospital based study. Ethiop J Health Dev. 2011[cited 2019 Jan 15]:25(1):17-21. Available from: https://www.ajol.info/index.php/ ejhd/article/view/69841/57922

19. Silva C, Pereira D, Almeida D, Venâncio M. Diabetic foot and assessment of the risk of ulceration. Rev Enferm Ref. 2014[cited 2019 Mar 11]: 4(1):153-61. Available from: http://www.scielo.mec.pt/pdf/ref/ vserIVnl/serIVnlal7.pdf

20. Vieira MA, Ohara CVS, De Domenico EBL. The construction and validation of an instrument for the assessment of graduates of undergraduate nursing courses. Rev. Latino-Am. Enfermagem. 2016[cited 2019 Mai 11]:24:e2710. Available from: http://www.scielo.br/ pdf/rlae/v24/pt_0104-1169-rlae-24-02710.pdf

21. Plassa BO, Alarcon MFS, Damaceno DG, Sponchiado VBY, Braccialli LAD, Silva JAVE, et al. Fluxograma descritor no atendimento à pessoa idosa vitima de violência: uma perspectiva interdisciplinar. Esc Anna Nery. 2018[cited 2019 Jan 22]:22(4):e20180021. Available from: http:// www.scielo.br/pdf/ean/v22n4/pt_1414-8145-ean-22-04-e20180021.pdf

22. Houts OS, Doak CC, Doak LG, Loscalzo MJ. The role of pictures in improving health communication: a review of research on attention, comprehension, recall, and adherence. Patient Educ Couns. 2006[cited 2019 Jan 22]:61(2):173-90. doi: https://doi.org/10.1016/j. pec.2005.05.004

23. Serxner S. How readability of material affects outcomes. $J$ Vasc Nurs. 2000[cited 2019 Jan 22]:18(3):97-101. doi: https://doi. org/10.1067/mvn.2000.109281

RECEBIDO: 31/07/2019 - ACEITO: 27/08/2019 